Bull. Austral. Math. Soc.

VoL. 40 (1989) [397-405]

\title{
WEIGHTED CONVOLUTIONS OF CERTAIN POLYNOMIALS
}

\author{
MASSOUD JAHANGIRI
}

For $\alpha \geqslant 0$ and $\beta \geqslant 0$, let $K(\alpha, \beta)$ consist of those functions $f(z)$, analytic and non-zero in $|z|<1$, such that for $\theta_{1}<\theta_{2}<\theta_{1}+2 \pi$ and $0<r<1,-\alpha \pi \leqslant \arg f\left(r e^{i \theta_{2}}\right)-$ $\arg f\left(r e^{i \theta_{1}}\right)+1 / 2(\alpha-\beta)\left(\theta_{1}-\theta_{2}\right) \leqslant \beta \pi$. It is conjectured that for $1 \leqslant \alpha \leqslant \beta$ and $\alpha$ an integer, the weighted convolution of polynomials having their zeros on $|z|=1$ and belonging to $K(\alpha, \beta)$, also belong to $K(\alpha, \beta)$. This conjecture is known to be true for the case $\alpha=1$, which leads to an alternative proof for the generalised Polya-Schoenberg conjecture. The case $\alpha=2$ is also known to be true for cubic polynomials. We prove the conjecture for certain quartic polynomials when $2 \leqslant \alpha \leqslant 4$.

\section{INTRODUCTION}

1.1. For $\alpha \geqslant 0$ and $\beta \geqslant 0$, let the Kaplan class $K(\alpha, \beta)$, be the class of functions $f(z)$, analytic and non-zero in $|z|<1$ such that for $\theta_{1}<\theta_{2}<\theta_{1}+2 \pi$, and $0<r<1$,

$$
-\alpha \pi \leqslant \arg f\left(r e^{i \theta_{2}}\right)-\arg f\left(r e^{i \theta_{1}}\right)+\frac{1}{2}(\alpha-\beta)\left(\theta_{1}-\theta_{2}\right) \leqslant \beta \pi .
$$

For $\alpha \geqslant 1$ and $\beta \geqslant 1$ where $\alpha$ is an integer, define

$$
Q_{n}(z ; \theta)=(1+z)^{\alpha-1} \prod_{j=1}^{n+1-\alpha}\left(1+z e^{i(2 j+\alpha-n-2) \theta}\right),
$$

where $\theta=\pi /(n+\beta-\alpha)$ and $1 \leqslant \alpha \leqslant n$.

Recall that the Hadamard product or convolution of two power series

$$
f(z)=\sum_{n=0}^{\infty} a_{n} z^{n} \text { and } g(z)=\sum_{n=0}^{\infty} b_{n} z^{n} \text { is }(f * g)(z)=\sum_{n=0}^{\infty} a_{n} b_{n} z^{n}
$$

Define $Q_{n}^{(-1)}(z ; \theta)$ so that $Q_{n}^{(-1)}(z ; \theta)+Q_{n}(z ; \theta)=1 /(1-z)$. The following is a special case of a conjecture given in [1].

Received 29 November 1988

This work was begun while the author was visiling the Universityof Kentucky at Lexington, where he enjoyed many valuable discussions with Professor Ted J. Suffridge.

Copyright Clearance Centre, Inc. Serial-lee code: 0004-9729/89 $\$ A 2.00+0.00$. 
1.2. Conjecture. Let $1 \leqslant \alpha \leqslant n$ and $\alpha \leqslant \beta$ where $\alpha$ and $n$ are integers. Assume that the polynomials

$$
p_{n}(z)=\prod_{k=1}^{n}\left(1+z e^{i \phi_{k}}\right) \text { and } q_{n}(z)=\prod_{k=1}^{n}\left(1+z e^{i \psi_{k}}\right)
$$

belong to $K(\alpha, \beta)$. Then the polynomial

$$
R_{n}(z)=\left(p_{n} * q_{n}\right)(z) * Q_{n}^{(-1)}(z ; \theta)
$$

also belongs to $K(\alpha, \beta)$.

This conjecture is proved for the case $\alpha=n-1=2$ in ([1, Theorem 4]) and for the case $1=\alpha \leqslant \beta$ by Suffridge ([10, Theorem 5$])$.

Since starlike functions of order $\gamma ; \gamma \leqslant 1$, can be characterised as the class of limits of sequences of polynomials in $K(\alpha, \alpha+2-2 \gamma) ; \alpha \geqslant 1$ (see [1, Theorem 2] and [10, Theorem 1]), the above conjecture for $\alpha=1$, leads to an alternative proof for the generalised Polya-Schoenberg conjecture [3]. The special cases $\gamma=0$ and $1 / 2$ were first proved by Ruscheweyh and Sheil-Small [6] while the general case $\gamma \leqslant 1$ was obtained by Suffridge [10], and later by Lewis [2] and Ruscheweyh [4]. The truth of our conjecture for other values of $\alpha$, that is $\alpha \neq 1$, may throw new light on a variety of old problems, because $K(\alpha, \beta)$ is closely related to several well-knnown classes of analytic and univalent functions. (For more details see [5-9]). In this note we prove the following

Theorem 1.3. Let $2 \leqslant \alpha \leqslant 4$ and $\alpha \leqslant \beta$ where $\alpha$ is an integer. Assume that the polynomials

$$
p_{4}(z)=\prod_{k=1}^{4}\left(1+z e^{i \phi_{k}}\right) \text { and } q_{4}(z)=\prod_{k=1}^{4}\left(1+z e^{i \psi_{k}}\right)
$$

belong to $K(\alpha, \beta)$ where $\phi_{k}=-\phi_{5-k}$ and $\psi_{k}=-\psi_{5-k}$. Then $R_{4}(z)=\left(p_{4} * q_{4}\right)(z) *$ $Q_{4}^{(-1)}(z ; \theta)$ also belongs to $K(\alpha, \beta)$.

Note that Theorem 1.3 is true for $\alpha=1$ by Suffridge ([10, Theorem 5]).

\section{SOME KEY LEMMAS}

To prove our theorem we shall need the following lemmas, the first of which is a special case of Theorem 1 in [1]. 
Lemma 2.1. Let $0 \leqslant \phi_{1} \leqslant \phi_{2} \leqslant \pi$. Then for $2 \leqslant \alpha \leqslant 4$ and $\alpha \leqslant \beta$, the polynomial

$$
p_{4}(z)=\left(1+z e^{i \phi_{1}}\right)\left(1+z e^{i \phi_{2}}\right)\left(1+z e^{i\left(2 \pi-\phi_{2}\right)}\right)\left(1+z e^{i\left(2 \pi-\phi_{1}\right)}\right)
$$

belongs to $K(\alpha, \beta)$ if and only if $0 \leqslant \phi_{1} \leqslant(\beta \pi) /(4+\beta-\alpha)$ and $((4-\alpha) \pi) /(4+\beta-\alpha) \leqslant \phi_{2} \leqslant \pi$.

LеммA 2.2. Let $0<c<1,-c \leqslant x \leqslant 1,-c \leqslant u \leqslant 1,-1 \leqslant y \leqslant c,-1 \leqslant v \leqslant c$, $y \leqslant x$ and $v \leqslant u$. Then

$$
F(x, y, u, v)=2+\frac{(x+y)^{2}(u+v)^{2}}{(1+c)^{2}}-\frac{2(1+2 x y)(1+2 u v)}{1+2 c} \geqslant 0 .
$$

Proof: The minimum of $F$ occurs either at a critical point or on the boundary. By setling $\partial F / \partial x=\partial F / \partial y=0$ we obtain either $1+2 u v=0$ or $\left((1+c)^{2}(1+2 u v)\right) /\left((1+2 c)(u+v)^{2}\right)=1$. By setting $\partial F / \partial u=\partial F / \partial v=0$ we obtain either $1+2 x y=0$ or

$\left((1+c)^{2}(1+2 x y)\right) /\left((1+2 c)(x+y)^{2}\right)=1$. If $1+2 u v=0$, then $F(x, y, u, v)=$ $2+\left((x+y)^{2}(u+v)^{2}\right) /\left((1+c)^{2}\right)>0$.

If $\left((1+c)^{2}(1+2 u v)\right) /\left((1+2 c)(u+v)^{2}\right)=1$, then $x=y$ and so $F(x, y, u, v)=$ $2\left\{1-(u+v)^{2} /(1+c)^{2}\right\} \geqslant 0$.

Similarly, $F(x, y, u, v) \geqslant 0$ when $1+2 x y=0$ or $(1+c)^{2}(1+2 x y)=(1+2 c)(x+y)^{2}$.

Now we check the values of $F(x, y, u, v)$ on the boundary. Since $F(x, y, u, v)$ is symmetric in $(x, y)$ and $(u, v)$, it would be enough to show that $F(x, y, u, v) \geqslant 0$ on the boundary involving $x$ and $y$. Let $y=-1$. Then

$$
\begin{gathered}
F(x,-1, u, v)=\frac{(u+v)^{2}}{(1+c)^{2}} x^{2}+2\left\{\frac{2(1+2 u v)}{1+2 c}-\frac{(u+v)^{2}}{(1+c)^{2}}\right\} x+2 \\
\quad+\frac{(u+v)^{2}}{(1+c)^{2}}-\frac{2(1+2 u v)}{1+2 c} .
\end{gathered}
$$

Setting $d F(x,-1, u, v) / d x=0$ we obtain $x_{0}=1-2(1+c)^{2}(1+2 u v) /(1+2 c)(u+v)^{2}$. But $F\left(x_{0},-1, u, v\right)=2\left\{1+(1+2 u v) /(1+2 c) x_{0}\right\} \geqslant 0$ because we must have $-c \leqslant$ $x_{0} \leqslant 1$. Also observe that $F(-c,-1, u, v)=(u-v)^{2} \geqslant 0$ and $F(1,-1, u, v)=2\{1+$ $(1+2 u v) /(1+2 c)\} \geqslant 0$. Then $F(x, y, u, v) \geqslant 0$ when $y=-1$. If $x=1$. then $r(1, y, u, v)=F(-y,-1, u, v) \geqslant 0$. Let $y=c$. Then

$$
\begin{aligned}
F(x, c, u, v)= & \frac{(u+v)^{2}}{(1+c)^{2}} x^{2}+2 c\left\{\frac{(u+v)^{2}}{(1+c)^{2}}-\frac{2(1+2 u v)}{1+2 c}\right\} x+2 \\
& +\frac{c^{2}(u+v)^{2}}{(1+c)^{2}}-\frac{2(1+2 u v)}{1+2 c} .
\end{aligned}
$$


Letting $d F^{\prime}(x, c, u, v) / d x=0$ we obtain $x_{1}=c\left\{\left(2(1+c)^{2}(1+2 u v)\right) /\left((1+2 c)(u+v)^{2}\right)\right.$ $-1\}$.

Observe that $F\left(x_{1}, c, u, v\right) \geqslant 0$ because we must have $y=c \leqslant x_{1} \leqslant 1$. If $x=-c$, then by setting $d F(-c, y, u, v) / d y=0$ we obtain $y_{1}=-x_{1}$, and so $F\left(-c, y_{1}, u, v\right)=$ $F\left(x_{1}, c, u, v\right) \geqslant 0$. If $x=y$, then it is easy to see that

$$
F(x, x, u, v)=2\left\{2\left[\frac{(u+v)^{2}}{(1+c)^{2}}-\frac{1+2 u v}{1+2 c}\right] x^{2}+1-\frac{1+2 u v}{1+2 c}\right\} \geqslant 0 .
$$

Therefore $F(x, y, u, v)$ is never negative for the given $x, y, u, v$ and $c$.

The following Lemma can also be proved using a similar argument and so we omit its proof.

LemMA 2.3. Let $0<c<1,-c \leqslant x \leqslant 1,-c \leqslant u \leqslant 1,-1 \leqslant y \leqslant c,-1 \leqslant v \leqslant c$, $y \leqslant x$ and $v \leqslant u$. Then

$$
F(x, y, u, v)=1+\frac{(1+2 x y)(1+2 u v)}{1+2 c}-\frac{2(x+y)(u+v)}{1+c} \geqslant 0 .
$$

LEMMA 2.4. Let $0<c<1,-c \leqslant x \leqslant 1,-c \leqslant u \leqslant 1,-1 \leqslant y \leqslant c, 1 \leqslant v \leqslant c$, $y \leqslant x$ and $v \leqslant u$. Furthermore, let

$$
-(1+c) \leqslant \frac{(x+y)(u+v)}{1+c}<-2 c
$$

and

$$
1+2 c^{2}<\frac{(1+2 x y)(1+2 u v)}{1+2 c} \leqslant 1+2 c
$$

Then

$$
F(x, y, u, v)=\frac{(1+2 x y)(1+2 u v)}{1+2 c}+\frac{2 c(x+y)(u+v)}{1+c}+2 c^{2}-1 \leqslant 0 .
$$

Proof: Since $\left(\partial^{2} F / \partial x^{2}\right)\left(\partial^{2} F / \partial y^{2}\right)-\left(\partial^{2} F / \partial x \partial y\right)^{2}=-\left(\partial^{2} F / \partial x \partial y\right)^{2}<0$, the maximum of $F(x, y, u, v)$ occurs on the boundary. Note that $\partial^{2} F / \partial x \partial y$ cannot be equal to zero, because that contradicts (2.4.2). Observe that $F(x, y, u, v)$ is symmetric in $(x, y)$ and $(u, v)$. Therefore it would be enough to show that $F(x, y, u, v) \leqslant 0$ for the boundary values involving $x$ and $y$. Since $(x+y)(u+v)<0$, withont loss of generality we assume that $x+y<0$ and $u+v>0$.

(i) If $x=-c$, then

$$
\begin{aligned}
& F(-c, y, u, v)=2 c\left\{\frac{u+v}{1+c}-\frac{1+2 u v}{1+2 c}\right\} y+\frac{1+2 u v}{1+2 c}-\frac{2 c^{2}(u+v)}{1+c}+2 c^{2}-1, \\
& \text { where, by }(2.4 .1),-1 \leqslant y \leqslant-c .
\end{aligned}
$$


Now we check $F(-c, y, u, v)$ for $y=-c$ and $y=-1$.

(i)(1) Let $y=-c$. Then

$$
F(-c,-c, u, v)=\frac{-4 c^{2}(u+v)}{1+c}+\frac{\left(1+2 c^{2}\right)(1+2 u v)}{1+2 c}+2 c^{2}-1 \leqslant 0,
$$

because $1+2 u v \leqslant 1+2 c$ and for $x=y=-c,-2 c(u+v)<-2 c(1+c)$, by (2.4.1).

(i)(2) Let $y=-1$. Then

$$
F(-c,-1, u, v)=-2 c(u+v)+\frac{2 c(1+2 u v)}{1+2 c}+2 c^{2}-1
$$

For $x=-c$ and $y=-1$ we obtain from (2.4.1) and (2.4.2) that $2 c<u+v$ and $1+2 u v \leqslant 1+2 c$. Substituting these in $F(-c,-1, u, v)$, it follows that

$$
F(-c,-1, u, v) \leqslant-2 c^{2}+2 c-1 \leqslant 0
$$

(ii) If $x=1$, then (by (2.4.1)), $y=-1$ and $c=0$. Therefore

$$
F(1,-1, u, v)=-2-2 u v<0 .
$$

(iii) If $y=-1$, then

$$
F(x,-1, u, v)=2\left\{\frac{c(u+v)}{1+c}-\frac{1+2 u v}{1+2 c}\right\} x+\frac{1+2 u v}{1+2 c}-\frac{2 c(u+v)}{1+c}+2 c^{2}-1
$$

We check $F(x,-1, u, v)$ for $x=-c$ and $x=1$. By (i)(2), $F(-c,-1, u, v) \leqslant 0$ and by (ii), $F(1,-1, u, v) \leqslant 0$.

(iv) If $y=+c$, then (by (2.4.1)), $x=-c=0$ and $u=v=0$. Therefore

$$
F(-c, c, u, v) \leqslant 0 \text { when } c=0 .
$$

Thus $F(x, y, u, v)$ is never positive.

LEMMA 2.5. If we change $x$ to $-y$ and $y$ to $-x$ or change $u$ to $-v$ and $v$ to $-u$ in Lemma 2.4, we obtain

$$
\frac{(1+2 x y)(1+2 u v)}{1+2 c}-\frac{2 c(x+y)(u+v)}{1+c}+2 c^{2}-1 \leqslant 0
$$


LEMMA 2.6. The polynomial $R_{4}(z)$ in Theorem 1.3 , has all its zeros on $|z|=1$.

Proof: Using trigonometric identities we can write

$$
Q_{4}(z ; \theta)=1+2(1+\cos \phi) z+2(1+2 \cos \phi) z^{2}+2(1+\cos \phi) z^{3}+z^{4}
$$

where $\phi=(4-\alpha) \theta=[(4-\alpha) \pi] /(4+\beta-\alpha)$. Now

$$
R_{4}(z)=1+2 A z+B z^{2}+2 A z^{3}+z^{4}
$$

where $A=(x+y)(u+v) /(1+c), B=2(1+2 x y)(1+2 u v) /(1+2 c), 0<c=\cos \phi<$ $1,-c \leqslant x=\cos \phi_{1} \leqslant 1,-c \leqslant u=\cos \psi_{1} \leqslant 1,-1 \leqslant y=\cos \phi_{2} \leqslant c,-1 \leqslant v=$ $\cos \psi_{2} \leqslant c, y \leqslant x$ and $v \leqslant u$.

If $R_{4}(z)$ has zero in $|z|<1$, it must have a zero in $|z|>1$, and vice-versa. Therefore, to prove that $R_{4}(z)$ has all its zeros on $|z|=1$, it is sufficient to show that $R_{4}(z) \neq 0$ in $|z|<1$. Write $R_{4}(z)=\left(z^{2}+\alpha_{1} z+1\right)\left(z^{2}+\alpha_{2} z+1\right)=p(z) q(z)$ where $\alpha_{1}=A-\sqrt{A^{2}+2-B}$ and $\alpha_{2}=A+\sqrt{A^{2}+2-B}$. By Lemma 2.2., $A^{2}+2-B \geqslant 0$, so $\alpha_{1}$ and $\alpha_{2}$ are real. Now we need to show that $p(z) \neq 0$ and $q(z) \neq 0$ in $|z|<1$. For this, it is sufficient to show that $-2 \leqslant \alpha_{1}$ and $\alpha_{2} \leqslant 2$ since $\alpha_{1} \leqslant \alpha_{2}$.

For the first case, we observe that $-2 \leqslant \alpha_{1}$ because $-2 \leqslant A-\sqrt{A^{2}+2-B}$ if and only if $2+B+4 A \geqslant 0$, which is true by Lemma 2.3. For the second case, we observe that $\alpha_{2} \leqslant 2$ because $2+B-4 A \geqslant 0$ by Lemma 2.3. This completes the proof.

\section{Proof of 'TheOREM 1.3}

3.1. By Lemma 2.6, $R_{4}(z)$ has all its zeros on $|z|=1$ and can be written as $R_{4}(z)=$ $1+2 A z+B z^{2}+2 A z^{3}+z^{4}$ where $A$ and $B$ are as in (2.6.1). Then there exist $\theta_{1}$ and $\theta_{2}$ with $0 \leqslant \theta_{1} \leqslant \theta_{2} \leqslant \pi$ such that

$$
R_{4}(z)=1+2\left(\cos \theta_{1}+\cos \theta_{2}\right) z+2\left(1+2 \cos \theta_{1} \cos \theta_{2}\right) z^{2}+2\left(\cos \theta_{1}+\cos \theta_{2}\right) z^{3}+z^{4}
$$

where

$$
A=\cos \theta_{1}+\cos \theta_{2} \text { and } B=2\left(1+2 \cos \theta_{1} \cos \theta_{2}\right)
$$

Because $p_{4}(z)$ and $q_{4}(z)$ are in $K(\alpha, \beta)$, then (by Lemma 2.1),

$$
-(1+c) \leqslant x+y, u+v \leqslant 1+c \text { and }-1 \leqslant 1+2 x y, 1+2 u v \leqslant 1+2 c .
$$

So, by (2.6.1) and (3.1.1),

$$
-(1+c) \leqslant \cos \theta_{1}+\cos \theta_{2} \leqslant 1+c \text { and }-1 \leqslant 1+2 \cos \theta_{1} \cos \theta_{2} \leqslant 1+2 c .
$$

To show that $R_{4}(z)$ belongs to $K(\alpha, \beta)$, it is sufficient (by Lemma 2.1.), to show that $\theta_{1}$ is never greater than $\beta \pi /(4+\beta-\alpha)$ and $\theta_{2}$ is never less than $[(4-\alpha) \pi] /(4+\beta-\alpha)$. 
3.2. First we show that $\theta_{1}$ is always between 0 and $\beta \pi /(4+\beta-\alpha)$. Assume that $\theta_{1}=\{\beta \pi /(4+\beta-\alpha)\}+\varepsilon$ where $\varepsilon>0$. Then

$$
-1 \leqslant \cos \theta_{2} \leqslant \cos \theta_{1}<-c .
$$

From (3.1.3) and (3.2.1) it follows that

$$
-(1+c) \leqslant \cos \theta_{1}+\cos \theta_{2}<-2 c \text { and } 1+2 c^{2}<1+2 \cos \theta_{1} \cos \theta_{2} \leqslant 1+2 c
$$

Therefore, by (2.6.1) and (3.1.2),

$$
-(1+c)^{2} \leqslant(x+y)(u+v)<-2 c(1+c)
$$

and

$$
(1+2 c)\left(1+2 c^{2}\right)<(1+2 x y)(1+2 u v) \leqslant(1+2 c)^{2} .
$$

Note that the above two inequalities are the conditions (2.4.1) and (2.4.2).

Solving the first equation of (3.1.1) for $\cos \theta_{2}$ and substituting this into the second equation, we obtain $\cos ^{2} \theta_{1}-A \cos \theta_{1}+1 / 4 B-1 / 2=0$. Then

$$
\cos \theta_{1}=\frac{1}{2}\left\{A \pm \sqrt{A^{2}+2-B}\right\}
$$

Now from (3.2.1) and (3.2.2) it follows that

$$
-2 \leqslant 2 \cos \theta_{1}=A \pm \sqrt{A^{2}+2-B}<-2 c
$$

We will show that (3.2.3) is never true. If $A+\sqrt{A^{2}+2-B}<-2 c$, then $4 A c+B-$ $2+4 c^{2}>0$ which is not true (by Lemma 2.4.). If $\cos \theta_{1}=1 / 2\left(A-\sqrt{A^{2}+2-B}\right)<-c$, then by (3.1.1), $\cos \theta_{2}=A-\cos \theta_{1}=1 / 2\left(A+\sqrt{A^{2}+2-B}\right)$. Since $\cos \theta_{2}<-c$ (by (3.2.1)), $A+\sqrt{A^{2}+2-B}<-2 c$. But by Lemma 2.4, this cannot be true. Therefore we conclude that $\theta_{1}$ is never greater than $\beta \pi /(4+\beta-\alpha)$.

3.3. Finally, we show that $\theta_{2}$ is never less than $[(4-\alpha) \pi] /(4+\beta-\alpha)$. Suppose that $\theta_{2}=\{(4-\alpha) \pi /(4+\beta-\alpha)\}-\delta$ where $\delta>0$. Then

$$
c<\cos \theta_{2} \leqslant \cos \theta_{1} \leqslant 1
$$

From (3.1.1)-(3.1.3) and (3.3.1) we obtain

$$
\begin{gathered}
2 c(1+c)<(x+y)(u+v) \leqslant(1+c)^{2} \text { and } \\
(1+2 c)\left(1+2 c^{2}\right)<(1+2 x y)(1+2 u v) \leqslant(1+2 c)^{2}
\end{gathered}
$$


These are the conditions (2.4.1) and (2.4.2) if we change $x$ to $-y$ and $y$ to $-x$, or change $u$ to $-v$ and $v$ to $-u$.

Solving the first equation of (3.1.1) for $\cos \theta_{1}$ and substituting in the second one we obtain

$$
\cos \theta_{2}=\frac{1}{2}\left\{A \pm \sqrt{A^{2}+2-B}\right\}
$$

From (3.3.1) and (3.3.2) it follows that

$$
2 c<2 \cos \theta_{2}=A \pm \sqrt{A^{2}+2-B} \leqslant 2 .
$$

We will show that (3.3.3) is never true. If $2 c<A-\sqrt{A^{2}+2-B}$, then $B-4 A c+$ $4 c^{2}-2>0$. But this is impossible by Lemma 2.5 .

If $2 c<2 \cos \theta_{2}=A+\sqrt{A^{2}+2-B}$, then by (3.1.1) and (3.3.1),

$$
c<\cos \theta_{1}=A-\cos \theta_{2}=\frac{1}{2}\left\{A-\sqrt{A^{2}+2-B}\right\} .
$$

By Lemma 2.5, this is not possible. Therefore we conclude that $\theta_{2}$ is never less than $(4-\alpha) \pi /(4+\beta-\alpha)$. This completes the proof of Theorem 1.3.

\section{Conclusion}

Our intuition is that if $n$ is a sufficiently small positive integer, then for given polynomials $p_{n}(z)$ and $q_{n}(z)$ of the form

$$
p_{2 m}(z)=\prod_{k=1}^{2 m}\left(1+z e^{i \theta_{k}}\right) \text { or } p_{2 m+1}(z)=(1+z) p_{2 m}(z)
$$

where $\theta_{k}=-\theta_{2 m+1-k}$, an argument similar to that used to prove Theorem 1.3 can be used to show that if $p_{n}(z)$ and $q_{n}(z)$ belong to $K(\alpha, \beta)$ where $1 \leqslant \alpha \leqslant n$ and $\alpha \leqslant \beta$, then $R_{n}(z)=\left(p_{n} * q_{n}\right)(z) * Q_{n}^{(-1)}(z ; \theta)$ also belong to $K(\alpha, \beta)$. For example, when $n=5$, we obtain (from Theorem 1 of $[1]$ ) that $p_{5}(z)$ is in $K(2, \beta)$ if and only if $\pi /(3+\beta) \leqslant \theta_{1} \leqslant(1+\beta) /(3+\beta)$ and $3 \pi /(3+\beta) \leqslant \theta_{2} \leqslant \pi$. Now (analogous to Lemma 2.6) we may show that $R_{5}(z)$ has all its zeros on $|z|=1$. This means that $R_{5}(z)$ can be written in the form $p_{2 m+1}(z)$. Next (analogous to the proof of Theorem 1.3), we show that the zeros of $R_{5}(z)$ are located so that $R_{5}(z)$ belongs to $K(2, \beta)$.

For polynomials of large degree, the above method turns out to be lengthy and rather involved. Perhaps one can come up with a better technique which works for polynomials of any degree. A reader interested in pursuing this problem may find the studies in [5-10] and specially [1] (Section 4, p.56) of some use. 


\section{References}

[1] M. Jahangiri, 'A gap condition for the zeros of certain polynomials in Kaplan classes $K(\alpha, \beta)$ ', Mathematika 34 (1987), 53-63.

[2] J.L. Lewis, 'Convolutions of Starlike functions', Indiana Univ. Math. J. 27 (1978), 671-688.

[3] G. Polya and I.J. Schoenberg, 'Remarks on de la Vallee Poussin Means and convex conformal maps of the circle', Pacific J. Math. 8 (1958), 295-334.

[4] St. Ruscheweyh, 'Linear operators between classes of prestarlike functions', Comment. Math. Helv. 52 (1977), 497-509.

[5] St. Ruscheweyh, Convolutions in geometric function theory: Sem. Math. Sup. (L Presses, De L'Universile De Montréal, Montreal, Que., 1982).

[B] St. Ruscheweyh and T.B. Sheil-Small, 'Hadamard products of Schlicht functions and the Polya-Schoenberg conjecture', Comment. Math. Helv. 48 (1973), 119-135.

[7] T.B. Sheil-Small, 'The Hadamard product and linear transofrmations of classes of analytic functions', J. Analyse Math. 34 (1978), 204-239.

[8] T.B. Sheil--Small, 'Coefficients and integral means of some classes of analytic functions', Proc. Amer. Math. Soc. 88 (1983), 275-282.

[8] 'T.B. Sheil-Small, 'Some remarks on Bazilevic functions', J. Analyse Math. 43 (1983/84), 1-11.

[10] T.J. Suffridge, 'Starlike functions as limits of polynomials': Advances in Complex Function Theory. : Lecture Notes in Math 505 (Springer-Verlag, New York). pp. 164-203.

Department of Mathematica

University of California

Davis CA 95616

United States of America 